\title{
Extreme Ultraviolet Spectroscopy of Magnetic Cataclysmic Variables
}

\author{
FRITS PAERELS, ${ }^{1}$ MIN YOUNG HUR, 1 \\ AND CHRISTOPHER W. MAUCHE ${ }^{2}$ \\ ${ }^{1}$ Space Sciences Laboratory and Department of Physics, \\ University of California, Berkeley, CA 94720-7300 \\ ${ }^{2}$ Lawrence Livermore National Laboratory, \\ L-41, P.O. Box 808, Livermore, CA 94550.
}

\begin{abstract}
A longstanding problem in the interpretation of the X-ray and extreme ultraviolet emission from strongly magnetic cataclysmic variables can be addressed definitively with high resolution EUV spectroscopy. A detailed photospheric spectrum of the accretion-heated polar cap of the white dwarf is sensitive in principle to the temperature structure of the atmosphere. This may allow us to determine where and how the bulk of the accretion energy is thermalized. The EUVE data on AM Herculis and EF Eridani are presented and discussed in this context.
\end{abstract}

\section{Introduction}

When matter accretes onto a white dwarf star with a strong $\left(B>10^{7} \mathrm{G}\right)$ magnetic field, the accretion flow near the white dwarf will be dominated by the field geometry, and quasi-1D "column" accretion onto a small polar cap ensues. Such is the case in the "polar" (after the strong polarization of their optical emission), or AM Herculis subclass of cataclysmic variables (CVs). In these objects, the field is known to be strong enough to actually dominate the accretion flow all across the binary, and an accretion disk does not form (see Cropper 1990 for a general review). Spin-orbit synchronization seems to have taken place in all but a few of the members of the class.

Since particles falling freely onto a white dwarf do not acquire enough kinetic energy to penetrate the stellar atmosphere, the bulk of the available kinetic energy must be thermalized and radiated above the atmosphere under optically thin conditions (see Frank, King, \& Raine 1990 for a general treatment of accretion physics). In a simple model, the supersonically falling accretion flow therefore encounters a strong shock just above the stellar surface, at which the gas is heated to roughly the virial temperature $k T \sim G M m_{p} / R \sim 100 \mathrm{keV}$, with $M$ and $R$ the stellar mass and radius, and $m_{p}$ the proton mass. The shock-heated gas cools by hard X-ray emission, roughly half of which strikes the stellar surface. The hard X-ray irradiation heats the atmosphere to soft Xray/EUV emitting temperatures ( $k T \sim$ tens of $\mathrm{eV}$ ), as is easily shown by a blackbody argument applied to an estimate of the size of the accretion-heated polar cap. The polars should be naturally strong EUV sources.

The same simple argument also predicts that the observed hard and soft X-ray luminosities should be roughly comparable. But while the $\mathrm{X}$-ray spectral characteristics of AM Her stars generally conform to the predictions of the simple model outlined above, the luminosity balance does not. In most objects, there appears to be a large relative overluminosity in soft X-rays/EUV. In AM Her, the first discovered and brightest polar, this problem was already found with $H E A O-1$ (Rothschild et al. . 1981). The most recent and most convincing demonstration of the problem is provided by a large homogeneous dataset on numerous polars obtained with the ROSAT PSPC (Beuermann \& Schwope 1993). 


\section{The Radiative Energy Balance and EUV Spectroscopy}

Numerous suggestions have been made as to the cause of the overluminosity in the photospheric radiation. The interesting point here is that each of the various mechanisms implies a detectable effect on the temperature structure of the atmosphere, depending on where and how the bulk of the accretion energy is thermalized. This suggests that high-resolution spectroscopy of the white dwarf photosphere in the EUV band, where the energy distribution peaks, should hold the key to a solution of the "soft X-ray (or EUV) problem." A brief overview of the various suggested mechanisms follows.

The simplest idea is that steady nuclear burning supplies a large fraction of the soft X-ray luminosity - for white dwarfs, the efficiency of nuclear burning for converting mass to energy is much larger than the maximum accretion conversion energy. Existing calculations (Papaloizou, Pringle, \& McDonald 1982) indicate, however, that the burning would not be stable for the mass-transfer rates and stellar masses appropriate to AM Her stars.

Kuijpers \& Pringle (1982) suggested that dense blobs in the accretion stream would avoid being shocked above the stellar surface if their density contrast is high enough. Instead, they would penetrate to large optical depths into the atmosphere, and deposit their kinetic energy at large optical depths. Since the thermalization occurs at such large depths, the temperature structure of the atmosphere should look like that of an "ordinary" atmosphere with no external heat source. In a further development of this idea however, it has been found that the blobs probably create big "splashes" and may thermalize a significant fraction of their kinetic energy under optically thin conditions (Hameury \& King 1988). In that case, the atmosphere, and hence its emission spectrum, should bear the signature of the presence of this external heat source at small optical depths.

The simple calculations for the energy balance of the shocked gas assume instantaneous collisional coupling between ions and electrons. More recently, the structure of the accretion shock has been examined in more detail (Thompson \& Cawthorne 1987; Woelk \& Beuermann 1992). The length scale associated with energy exchange between ions and electrons can be a significant fraction of the shock height, and a large fraction of the accretion energy can be radiated away across the finite shock thickness if cyclotron cooling is important. This description of the accretion region is valid only if collective effects do not effectively increase the coupling between ions and electrons. Since optically thick cyclotron cooling dominates the radiative energy balance, the electron temperature remains at a fraction of the virial temperature, and the hard bremsstrahlung emission is suppressed.

Woelk \& Beuermann (1992) have performed detailed calculations for the accretion region. Their calculation shows that given the right conditions (density in the accretion stream and surface magnetic field strength) the electron temperature in the accretion region does indeed remain relatively low ( $\sim$ few $\mathrm{keV}$ ), and that the hard X-ray emission is indeed suppressed. The idea is consistent with a correlation observed between the soft/hard flux ratio, and the surface magnetic field strength of the white dwarf: objects with stronger magnetic fields have relatively larger soft/hard ratios, implying more efficient cyclotron cooling (Beuermann \& Schwope 1993). The one notable exception to this trend is AM Her-with a low surface field strength of only 15 MG (Schmidt, Stockman, \& Margon 1981; Latham, Liebert, \& Steiner 1981) it nevertheless has one of the largest soft/hard flux ratios.

Here again, there is a heat source at small enough optical depth such that one expects 
a signature in the photospheric EUV spectrum, which may allow for an independent verification of this explanation for the observed soft/hard flux ratios.

Finally, we point out that an X-ray heated atmosphere, as is present in the "classical" model for the EUV emission from polars, will also bear the mark of heating at small optical depth. In the next section, we will briefly discuss a calculation for the spectrum of an irradiated atmosphere, as an example of the diagnostic power of the photospheric EUV spectrum in polars.

\section{EUV Emission from X-ray Irradiated Atmospheres}

Any external source of heat in the white dwarf atmosphere, provided it is at sufficiently small depth, will produce a temperature and ionization structure that is very different from the structure of an "ordinary" atmosphere. Specifically, the temperature distribution will be much flatter in the outer parts of the atmosphere, and a temperature inversion will develop in the transition region to the optically thin, hot parts of the accretion region. Assuming LTE for the moment, these properties will qualitatively lead to a larger range of ionization states of a given element being visible in the photospheric spectrum, as compared to the spectrum of an ordinary atmosphere, and a reduced contrast at the absorption edges due to the flat temperature structure. The increased ionization and reduced opacity will also produce a relatively harder spectrum. The temperature inversion may produce a characteristic angular intensity pattern as a function of frequency that would reveal itself in phase-resolved spectroscopy of the accretion spot as limb darkening and brightening. Finally, at the top of the atmosphere in the transition region and above, the conditions are such that, at the right viewing angle, emission features may develop.

All these features are present in calculations for hard X-ray irradiated atmospheres performed by van Teeseling, Heise, \& Paerels (1994). These are LTE calculations, for an atmosphere of cosmic abundances (the accreting material maintains a steady supply of metals into the photosphere). The irradiation effect is incorporated by specifying the irradiating radiation field as the upper boundary condition, and solving the transfer equation. In Figure 1, we show an example of the spectrum of an atmosphere at $T_{\text {eff }}=$ $240,000 \mathrm{~K}, \log g=8$, with and without irradiation by hard thermal bremsstrahlung.

Calculations for the spectrum of the accretion region cooling by optically thick cyclotron emission (Woelk \& Beuermann 1992) generally show the same qualitative atmospheric structure. So far, models have been computed for pure hydrogen atmospheres only, which yield roughly the correct optical/UV continuum spectrum and the cyclotron emission spectrum. At higher energies, the opacity is dominated by highly ionized metals, whose presence completely dominates the shape of the EUV/soft X-ray spectrum. Hence, these models cannot yet be directly compared with EUV spectroscopic observational data.

\section{EUV/Soft X-ray Emission from AM Her}

As mentioned before, the brightest polar, AM Herculis, has a very large soft/hard flux ratio. Its low surface magnetic field strength of $\sim 15 \mathrm{MG}$ probably indicates that this large flux ratio cannot be explained by including the effects of cyclotron cooling on the structure of the accretion region. Nevertheless, the emission spectrum shows definite signs of external heating of some sort, as was first found in spectroscopic observations with the diffraction grating spectrometers on EXOSAT (Paerels, Heise, \& van Teeseling 1994). The white dwarf photospheric emission is definitely much harder than predicted for an ordinary atmosphere. In addition, there was evidence for the presence of absorption 


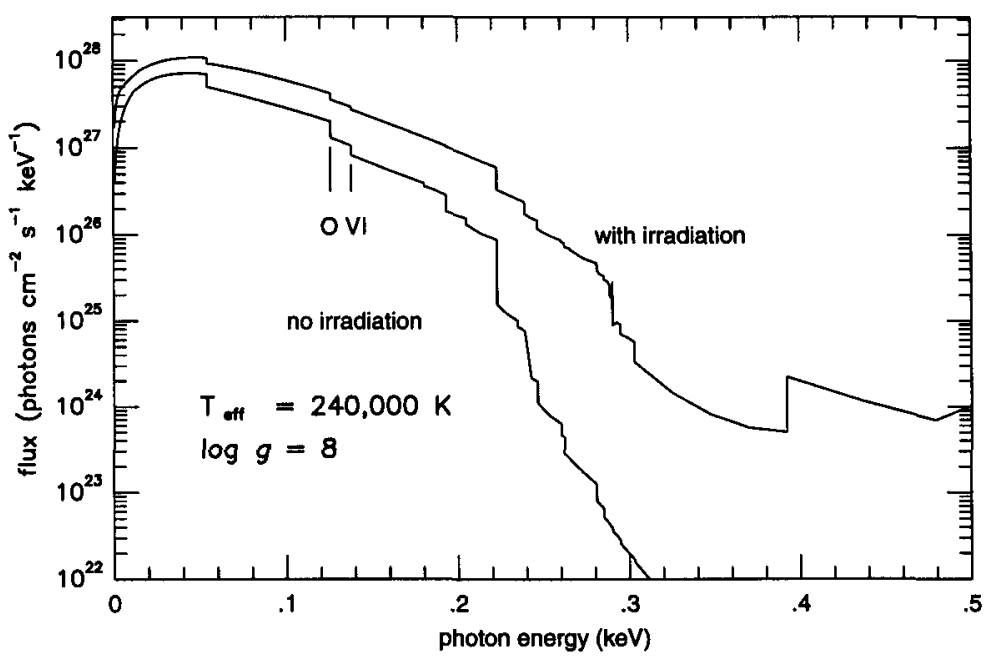

FIGURE 1. Flux spectrum of an atmosphere of cosmic abundances at $T_{\text {eff }}=240,000 \mathrm{~K}, \log g=8$. The lower curve is the spectrum for an atmosphere without irradiation, while the upper curve is for the same atmosphere with hard X-ray irradiation. The position of the O VI $2 s, 2 p$ edges has been indicated. Note the generally reduced contrast at absorption edges and the harder spectral shape for the irradiated atmosphere.

edges due to a wide range of ionization stages of oxygen and neon; this is illustrated in Figure 2, where we show the orbital average spectrum of AM Her as observed with EXOSAT in 1983.

The EXOSAT data had only moderate sensitivity to absorption features-the spectral resolution was about $3 \AA$ at $100 \AA$ with the high dispersion grating. Instead, these data cover a wide energy band $(0.1-2 \mathrm{keV})$. This source therefore was a natural target for observations with $E U V E$, to obtain high resolution spectroscopy over a narrower band.

We observed AM Her with EUVE for a total of $102,000 \mathrm{~s}$ effective exposure time over the period 1993 September 23-28, covering a total of 36.9 binary orbits of $3.09 \mathrm{hrs}$ each (Paerels et al. . 1995). We detected the source in the 75-120 $\AA$ band. The orbital average spectrum is shown in Figure 3 . We positively detect the Ne VI ground state and first excited state absorption edges at $78.5,85.3 \AA$. In addition, we detect a number of absorption lines which can be identified with a series of $n=2-3,4$ transitions in Ne VIII. The simultaneous presence of these two ions with very different ionization potentials ( $\chi=158,239 \mathrm{eV}$, respectively) in the EUV photosphere is qualitatively consistent with external heating of the atmosphere.

Surprisingly, there is no evidence for the O VI $2 s, 2 p$ edges at $89.8,98.3 \AA$. This is again qualitatively consistent with heating at small depths, which tends to reduce the contrast at absorption edges (cf., $\S 3$ ). However, it remains puzzling why there should be Ne VI absorption, since O VI and Ne VI have similar ionization potentials. An anomalously low oxygen abundance in the accreting gas seems excluded by observations of strong $\mathrm{O}$ 
AM Her 1983/222 EXOSAT TGS

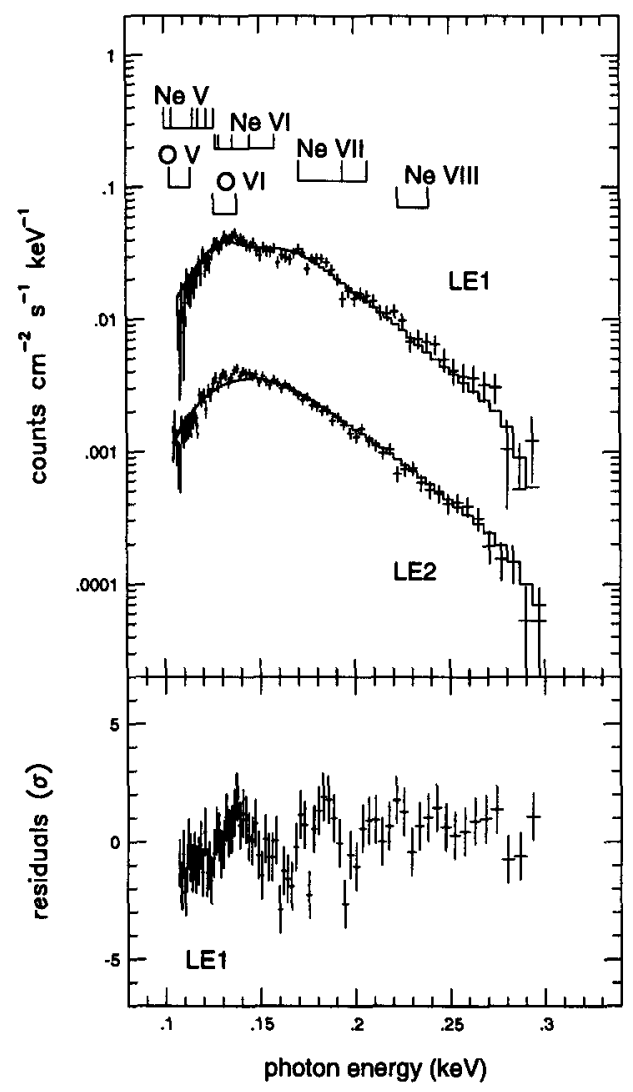

FIGURE 2. Orbital average photospheric spectrum of AM Her as observed with the EXOSAT Transmission Grating Spectrometers, with the high dispersion (LE1) and low dispersion (LE2) gratings. The solid line is a simple blackbody fit at $k T_{\mathrm{BB}}=28 \mathrm{eV}, N_{\mathrm{H}}=6 \times 10^{19} \mathrm{~cm}^{-2}$. Residuals to the LE1 fit are shown in the lower panel. The positions of a number of absorption edges from highly ionized $\mathrm{O}$ and $\mathrm{Ne}$ has been indicated. Note the presence of discrete structure in the spectra and the residuals, and the fact that the spectrum extends all the way out to the C K edge (280 eV) (from Paerels, Heise, \& van Teeseling 1994).

line emission in the far UV (O VI $2 s-2 p$, Raymond et al. 1995) and the optical (O III Bowen fluorescence, Schachter et al. 1991), both arising in the photoionized accretion stream.

There is evidence for a slight hardening of the spectrum at those orbital phases when we view the accretion spot at very shallow angles. This may indicate the presence of a temperature inversion in the photosphere.

All these facts are qualitatively consistent with the spectrum arising in an X-ray ir- 


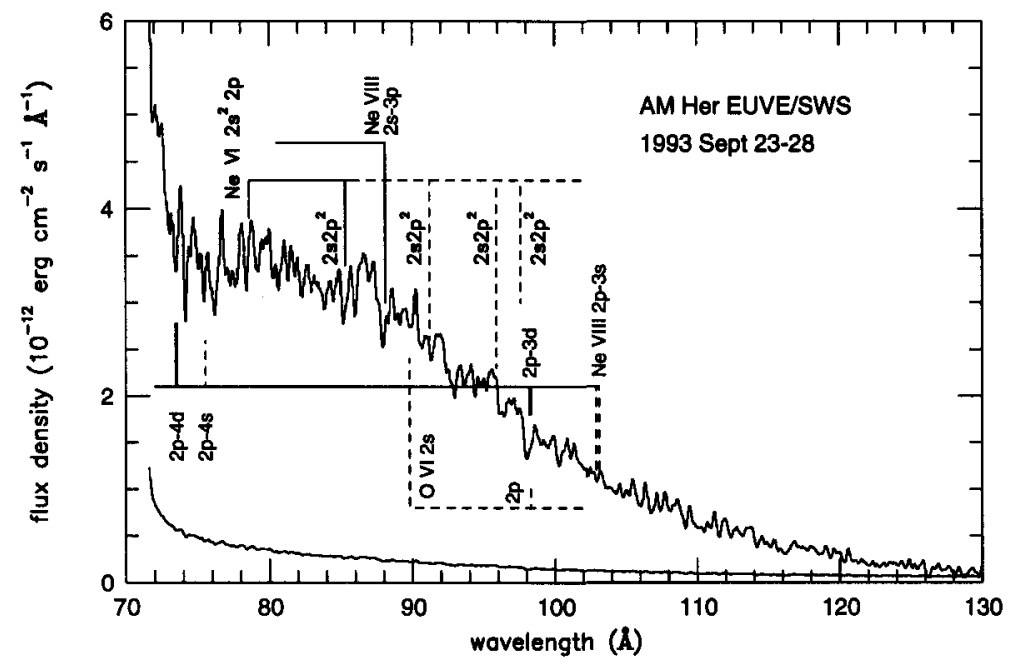

FIGURE 3. Orbital average spectrum of AM Her as observed with EUVE. The spectrum has been background subtracted; the lower curve represents the size of the error bars, based on counting statistics. We detect the Ne VI ground state and first excited state edges and Ne VIII $n=2-3,4$ absorption lines (indicated with solid lines above and below the spectrum).

radiated atmosphere. A detailed spectroscopic comparison with models for irradiated atmospheres to substantiate these suggestions is in preparation. Further details of the observation and the phase dependence of the spectrum may be found in Paerels et al. (1995).

\section{EUV Spectroscopy of EF Eri}

EF Eridani is another relatively bright polar. Contrary to AM Her, its soft/hard luminosity ratio is closer to that expected for a purely radiatively heated accretion spot (Beuermann, Stella, \& Patterson 1987; Beuermann \& Schwope 1993). In addition, on at least a number of occasions, the source also exhibited another feature predicted by the classical irradiation model: if the white dwarf atmosphere is heated by hard X-rays from the post-shock gas, any variability in hard X-rays should be tracked by the soft emission. Beuermann, Stella, \& Patterson (1987) found such behavior in Einstein IPC, data, although the correlation is absent in the recent ROSAT data (Beuermann, Thomas, \& Pietsch 1991).

We observed EF Eri with $E U V E$ for 80,500 s effective exposure time on 1993 September $5-9$. The orbital average spectrum is shown in Figure 4 . We detect a very strong $\mathrm{Ne}$ VI ground state absorption edge at $78 \AA$. There is evidence for an edge at $98 \AA$, which could be due to either O VI $2 p$ (but no ground state edge seen at $89 \AA$ ), or Ne V $2 s^{2} 2 p^{2}$ (ground state). Another edge near $\sim 113 \AA$ may be $\mathrm{Mg}$ IV $2 s^{2} 2 p^{5}$ (ground state). The spectrum is unfortunately too noisy to reveal any absorption lines. 


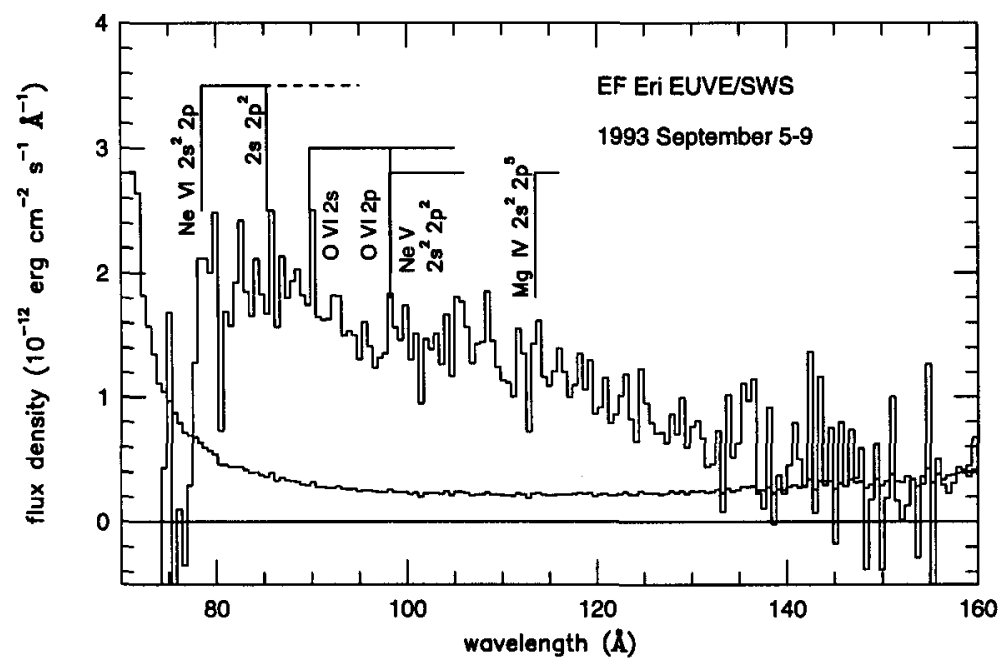

FIGURE 4. Orbital average spectrum of EF Eri as observed with EUVE. The spectrum has been background subtracted; the lower curve represents the size of the error bars, based on counting statistics. We detect the Ne VI ground state edge. There is evidence for either O VI $2 p$ or $\mathrm{Ne}$ $\mathrm{V}$ ground state absorption at $98 \AA$, and possibly Mg IV ground state absorption at $\sim 113 \AA$ (indicated with solid lines above the spectrum).

The presence of a strong Ne VI edge indicates lower ionization in EF Eri than in AM $\mathrm{Her}$, consistent with the detection of the lower ionization species $\mathrm{Ne} \mathrm{V}$ and $\mathrm{Mg} \mathrm{IV}$. It is tempting at this stage to interpret the presence of such a strong edge as evidence for the absence of irradiation (cf., $\S 3$ ), but in view of the limited bandwidth over which we observe the spectrum a detailed comparison with model atmospheres will be necessary to decide whether this suggestion is correct.

\section{Conclusions}

Detailed spectroscopy of the EUV spectrum of the accretion heated atmosphere of the white dwarf in magnetic CVs can help determine where and how the bulk of the accretion energy is thermalized. Spectroscopy with EUVE of AM Her and EF Eri confirms the potential of this diagnostic.

Preliminary investigation of the spectra of AM Her and EF Eri has already produced suggestive spectroscopic evidence for external heating of the white dwarf atmosphere in AM Her, while EF Eri may show the spectrum of an ordinary hot atmosphere, indicating perhaps that at the time of the $E U V E$ observation most of the accretion energy was being deposited at large optical depths.

Finally, we mention the fact that $E U V E$ observations of two other polars have also provided interesting spectroscopic information, although these objects are weaker, and the statistical quality of the spectra is somewhat limited. Vennes et al. (1995) report the 
detection of weak O VI absorption edges in the spectrum of VV Pup, and find that they need to assume an anomalously high oxygen abundance in the atmosphere in order to fit the spectrum, while Rosen et al. (this volume) detected Ne VI and Ne VIII absorption in RX J1938-461.

F.P. and M.Y.H. were supported by NASA grant NAG-5-2378. C.W.M.'s contribution to this work was performed under the auspices of the US Department of Energy by Lawrence Livermore National Laboratory under contract No. W-7405-Eng-48.

\section{REFERENCES}

Beuermann, K. Stella, L., \& PATterson, J. 1987, Einstein, observations of EF Eridani 2A 0311, the textbook example of AM Herculis type systems, ApJ, 316, 360

Beuermann, K., Thomas, H. -C., \& Petsch, W. 1991, Short time-scale X-ray variability in the AM Her type binary EF Eridani, A\&A, 246, L36

Beuermann, K, \& Schwope, A. D. 1993, AM Herculis Binaries, in Interacting Binary Stars, ed. A. W. Shafter, ASP Conf. Ser., 56, 119

Cropper, M. 1990, The polars, Space Sci. Rev, 54, 195

Frank, J., King, A., \& RaINe, D. 1990, Accretion power in astrophysics 2nd Ed., Cambridge University Press

HameURY, J. M., \& KING, A. R. 1988, The X-ray light curves of AM Herculis systems, A\&A, 235,433

KuiJPers, J., \& Pringle, J. E. 1982, Comments on radial white dwarf accretion, A\&A, 114, L4

Latham, D. W., LEbert, J., \& Steiner, J. E. 1981, The 1980, low state of AM Herculis, ApJ, 246, 919

Paerels, F., Heise, J., \& van Teeseling, A. 1994, Simultaneous soft and hard X-ray spectroscopy of AM Herculis with EXOSAT: discovery of photospheric absorption features, ApJ, 426, 313

Paenels, Hur, M. Y., Mauche, C. W., \& Heise, J. Extreme ultraviolet spectroscopy of the white dwarf photosphere in AM Herculis, ApJ, submitted

Papaloizou, J. C. B., Pringle, J. E., \& MCDonald, J. 1982, Steady nuclear burning on white dwarfs, MNRAS, 198, 215

RAYMond, J. C., MAUche, C. W., BowYER, S., \& HURwITZ, M. 1995, ORFEUS, observations of AM Herculis, ApJ, 440, 331

RotHSCHID, R. ET AL. 1981, The X-ray spectrum of AM Herculis from 0.1 to $150 \mathrm{keV}, \mathrm{ApJ}$, 250,723

Schachter, J., Filippenko, A. V., Kahn, S. M., \& Paerels, F. 1991, Bowen fluorescence in AM Herculis stars, ApJ, 373, 633

Schmmt, G. D., Stockman, H. S., \& Margon, B. 1981, A direct measurement of the magnetic field in AM Herculis, ApJ, 243, L157

Thompson, A. M., \& CAwTHORNe, T. V. 1987, Cyclotron emission from white dwarf accretion columns, MNRAS, 224, 425

van Teeseling, A., Heise, J., \& Paerels, F. 1994, X-ray irradiation of white dwarf atmospheres: the soft X-ray spectrum of AM Herculis, A\&A, 281, 119

VenNes, S., Szkody, P., Sion, E. M., \& Long, K. 1995, Extreme ultraviolet spectroscopy and photometry of VV Puppis during a high accretion state, ApJ, 445, in press

Woelk, U., \& BeuermanN, K. 1992, Particle heated atmospheres of magnetic white dwarfs, A\&A, 256, 498 